\title{
Prediction of gas-oil contact from surface seismic exploration
}

\author{
Yunosuke IWAKI $^{1}$, Hitoshi MIKADA ${ }^{2}$, Tada-nori GOTO ${ }^{2}$ and Junichi TAKEKAWA ${ }^{2}$ \\ ${ }^{1}$ Undergraduate School of Global Eng., Kyoto University \\ ${ }^{2}$ Dept. of Civil and Earth Res. Eng., Kyoto University
}

\begin{abstract}
The fluid behavior in the reservoir of hydrocarbon resources greatly affects the oil production. Quantifying the amount and the distribution of hydrocarbon in the subsurface offers useful information to modify the future planning. The gas-oil contact (GOC) is expected as an indicator of oil observed by surface seismic exploration at low frequency. In this study, we attempt to grasp the movement of GOC in a simulation based on the Biot equations. The Biot equations consider the poroelastic media composed by solid and fluid phase, which allow us to handle the fluid alteration directly. We simulated with a second-order, finite-difference Biot scheme and investigated the effect of GOC and oil-water contact (OWC) under some assumptions in fluid alteration. The results show the possibility of surface seismic to be used to estimate the behavior of GOC in the subsurface. We also introduce the concept of active/inactive pores to derive the property of solid matrix phase from solid grains and including fluid, which had little influence on the wave data received.
\end{abstract}

\section{INTRODUCTION}

Reservoir monitoring is beneficial to decide how to advance the oil production. The change inside the reservoir is observed through time-lapse 3D exploration methods that use both surface seismic and well-logging data. Since a well-logging method requires the suspension of production, the direct acquisition of fluid behavior by surface seismic method could help us determine the production plan in a simple and economical way.

In this paper, we focus on gas-oil contact (GOC) moving as an indicator of the fluid alternation from oil to gas in the oil production. Because the difference in the fluid physical properties between oil and gas is larger than that between oil and water, the movement of GOC is expected to be well observed compared to that of oil-water contact (OWC). We simulated the wave propagation in the reservoir modeled as a $2 \mathrm{D}$ medium to see if the movement of GOC/OWC could be estimated with the 2D finite-difference Biot method including the fluid effect in the pore space before and after the alteration. We confirmed that the detection of the change in OWC is much more difficult in surface seismic methods than that in GOC. We also investigated the partially fluid alteration in two cases.

\section{METHOD}

\section{(1) 2D finite-difference Biot model}

Biot introduced a description of wave- and pore-pressure fields in a porous rock $^{122}$. He considered two cases of the wave propagation with respect to the frequency range, but we think only of the low-frequency theory to deal with surface seismic.

In this paper, we use a second-order, finite-difference Biot scheme with staggered grids. The discretized equations derived from the Biot equations and from the velocity-stress formulation are introduced as in one ${ }^{3)}$ of the previous work.

\section{(2) Inclusion-based formulation}

In low-frequency Biot theory, the pore geometry is incorporated through the use of empirically determined macroscopic parameters so the effect of pore shape can't be considered. We use inclusion-based formulation, the extent method of high-frequency theory with complete fluid pressure communication $^{4)}$, etc. to accommodate pore geometry. The original formulation in high-frequency Biot theory assumes that all pores are isolated and doesn't satisfy the Biot-Gassmann theory which treats all pores are connected. However, the expanded theory can handle the pore shape effect without violating the Biot-Gassmann theory, and if we know the pore shape, we can describe the bulk modulus of the dry solid matrix, 
which is often given empirically. For simplicity of the calculation of inclusion-based formulation, we assume that each pore has a spherical shape and that the inclusion-based formulation follows the $T$ matrix approximation derived by Kuster and Toksöz theory ${ }^{5)}$.

\section{(3) Inactive porosity}

Biot theory for the wave propagation could be justified under the assumption that all pores are interconnected, and that isolated pores are included in the solid matrix. We call 'active pore' for the interconnected pores, while 'inactive pore' for the disconnected pores ${ }^{6}$. We apply the inclusion-based model again to consider the effect of the inactive pores that include fluid. This theory is defined for the high-frequency wave propagation method for a porous model without the communication in fluid pressure between pores, i.e. all pores are isolated. We use this theory to determine the bulk and sheer moduli of solid matrix with inactive pores before the wave propagation simulation. The simulation is done in low frequency with the model composed of fluid phase in active pore (with complete fluid pressure communication) and solid matrix (the mixture of solid grains and fluid in the inactive pores). In this paper, we follow the $T$ matrix approximation as the same way of the part (2).

\section{SIMULATION MODEL}

We use a simple simulation model as shown in Figure 1. The size of the model is $800 \mathrm{~m}$ in horizontal direction and $1000 \mathrm{~m}$ in depth. A water layer is placed in the upper part of the model with a depth of $350 \mathrm{~m}$, and the solid matrix under the seabed consists only of sandstone. We assume that there are only two types of fluid in the porous rock, initially separated at the depth $650 \mathrm{~m}$. Let us call 'area 1' for the upper part of the model and 'area 2' for the lower part filled with fluid different from the area 1. We apply Cerjan's absorbing boundary of a $100 \mathrm{~m}$ width to all the four edges. ${ }^{7)}$

The change in the depth of fluid contact is described as the alteration of fluid in the area passed by the descending contact of the two areas. The source is assumed as a Ricker wavelet with a center frequency of $45 \mathrm{~Hz}$ (adjusted the amplitude to be 1 ), whose source is placed at $300 \mathrm{~m}$ away from the left edge with a $200 \mathrm{~m}$ water depth of the water layer (shown as the circle in Figure 1). We set the receivers at a $250 \mathrm{~m}$ water depth with a $1 \mathrm{~m}$ interval between each pair of neighboring receivers. We get two sets of receiver data, one for the initial condition and the other for the case after the descent of the contact for $20 \mathrm{~m}$.
Table 1 Solid property (sandstone)

\begin{tabular}{lr}
\hline bulk modulus & $40 \mathrm{GPa}$ \\
sheer modulus & $0.82 \mathrm{GPa}$ \\
density & $2600 \mathrm{~kg} / \mathrm{m}^{3}$ \\
permeability & $10^{-12} \mathrm{~m}^{2}$ \\
porosity & $20 \%$
\end{tabular}

Table 2 Fluid property

\begin{tabular}{lrrr}
\hline & water & gas & oil \\
\hline $\begin{array}{l}\text { bulk modulus } \\
{[\mathrm{GPa}]}\end{array}$ & 2.25 & 0.00014 & 1.5 \\
$\begin{array}{l}\text { density } \\
{\left[\mathrm{kg} / \mathrm{m}^{3}\right]}\end{array}$ & 1000 & 1 & 850 \\
$\begin{array}{l}\text { viscosity } \\
{[\mathrm{Pa} \mathrm{s}]}\end{array}$ & 0.001 & 0.00002 & 1.726 \\
\hline
\end{tabular}

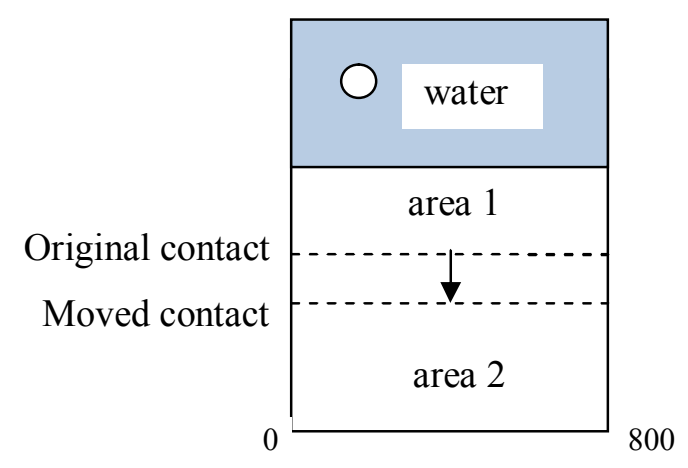

Figure 1 Simulation model

Finally, the solid and fluid properties are given in Tables 1 and 2.

\section{RESULT AND DISSCUSION}

In this section, we introduce three types of the fluid alteration in the areas 1 and 2 . In each type, we simulated for two cases: (a) area 1 is saturated by water and area 2 by oil (OWC moving) (b) area 1 is saturated by gas and area 2 by oil (GOC moving), and calculated the residual of before and after the event. 


\section{(1) Complete fluid alteration}

First, we assume that oil in the lower part of the reservoir is completely altered by gas or water as the contact moves down. The results are shown in Figure 2. The residual of case (b) is about ten times larger than case (a), which is caused by the difference of the fluid properties just above and below the contact, that is, gas properties are different from those of oil though the difference between water and oil properties is much smaller.
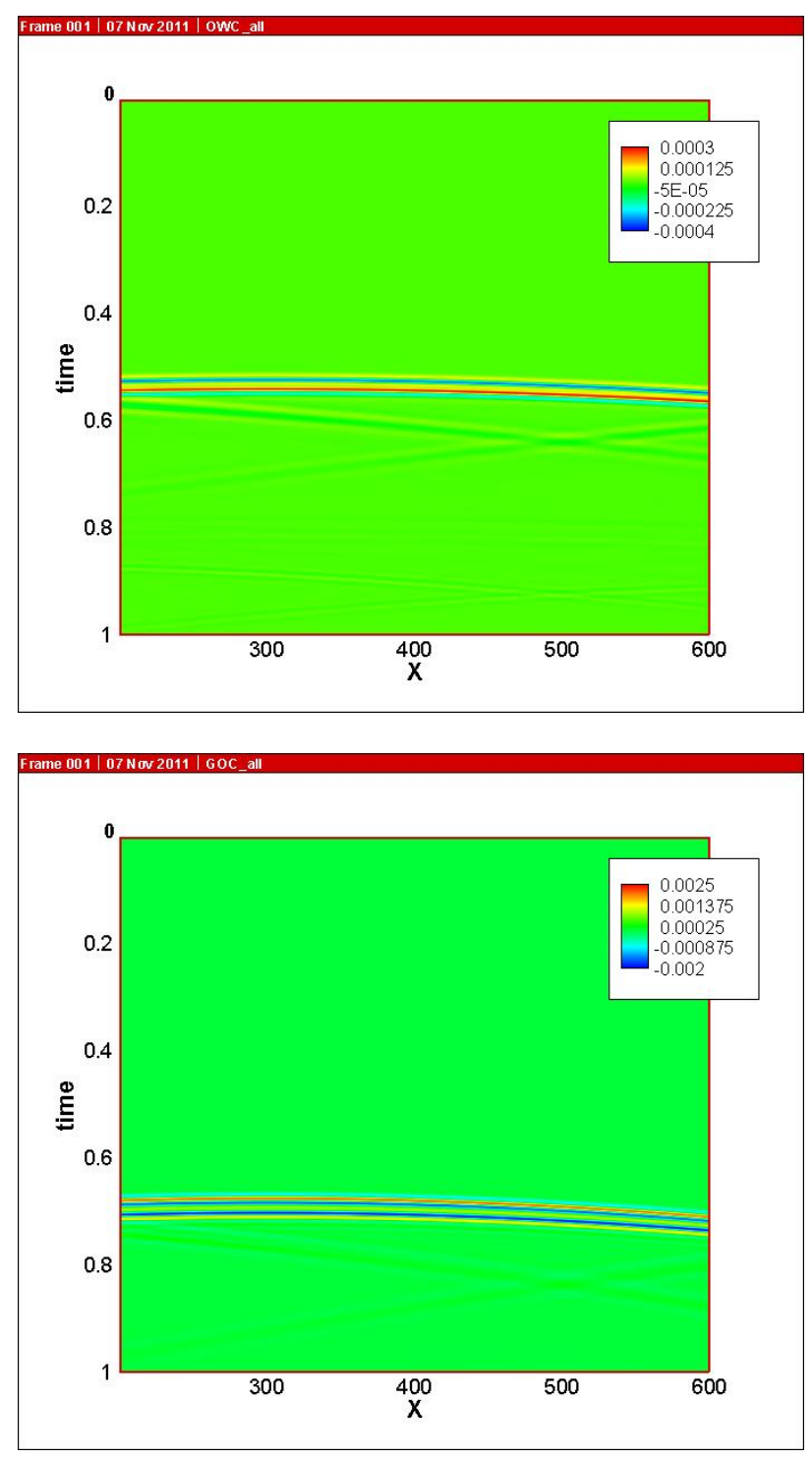

Figure 2 the residual of OWC (upper) and GOC (lower) for complete fluid change

\section{(2) Partial fluid alteration without inactive pores}

Next, we consider in the case that oil is partially ( $50 \%$ in the volume fraction) displaced by gas or water. The bulk modulus of fluid mixture is defined by Reuss average and the density by Voigt average. We apply the oil viscosity as the fluid mixture viscosity. As shown in Figure 3, the results are similar to the condition of complete fluid alteration in both cases.
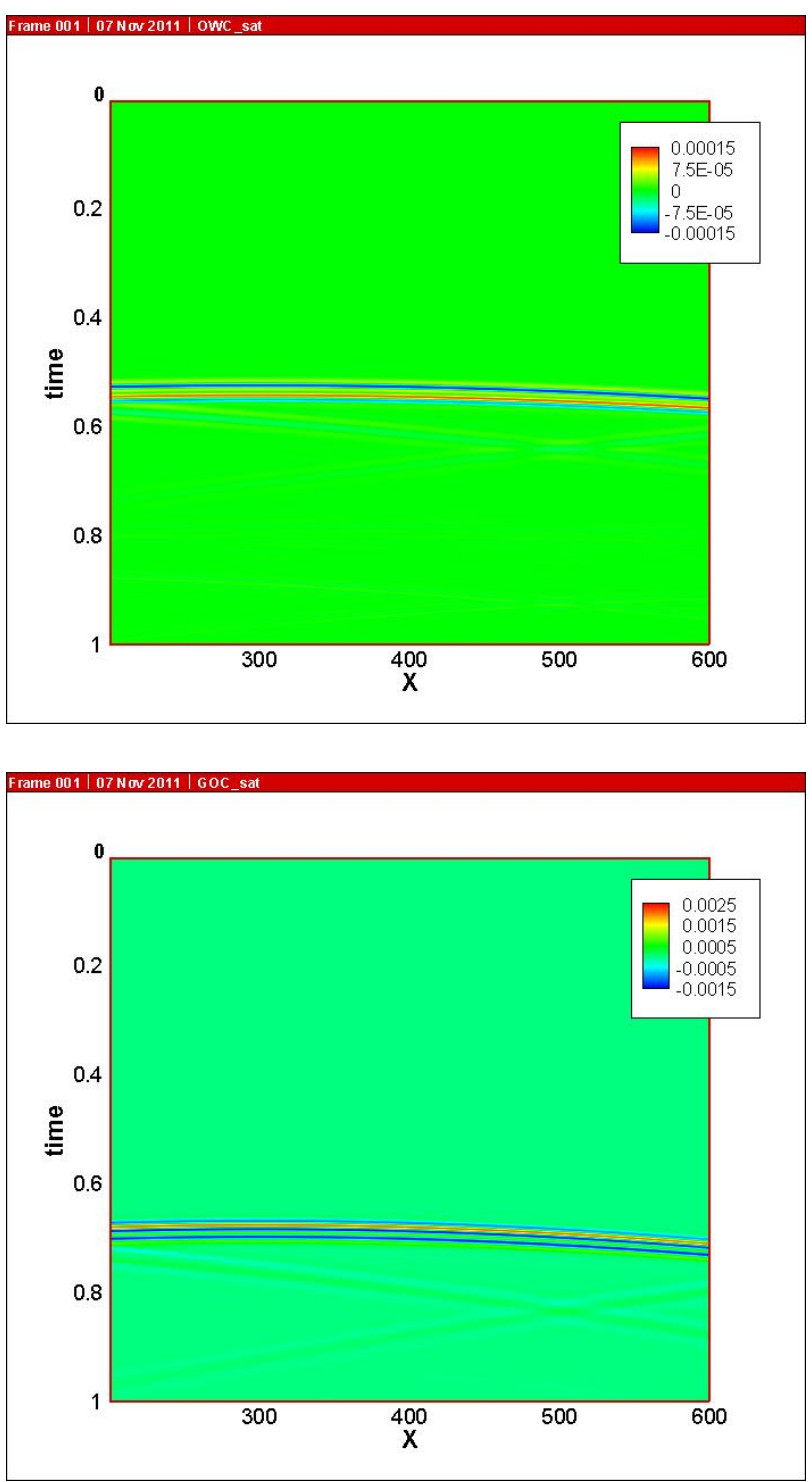

Figure 3 the residual of OWC (upper) and GOC (lower) for partial fluid change with volume fraction

\section{(3) Partial fluid alteration with inactive pores}

Finally, we adopt the active/inactive pore in the reservoir modeling (see section 2(3)). In this case, the fluid displacement occurs only in active pores and the fluid in inactive pores remains after the contact moving. To compare to the result of the case (2), the porosity ( $20 \%$ ) is divided in two; $10 \%$ for active porosity and $10 \%$ for inactive porosity, and the fluid in active pores is completely displaced. As shown in Figure 4, the residuals are the same order between the cases (2) and (3) for both OWC and GOC.

In this case, the properties of the solid matrix differ by the included fluid in contrast to the case of the partially fluid alteration without inactive pores, 
which uses the properties of sandstone shown in Table 1. Fluid parameters calculated by the method 2(3), however, become similar to those of sandstone independent on the include fluid. As a result, the two partially displacement cases (2) and (3) have few differences.
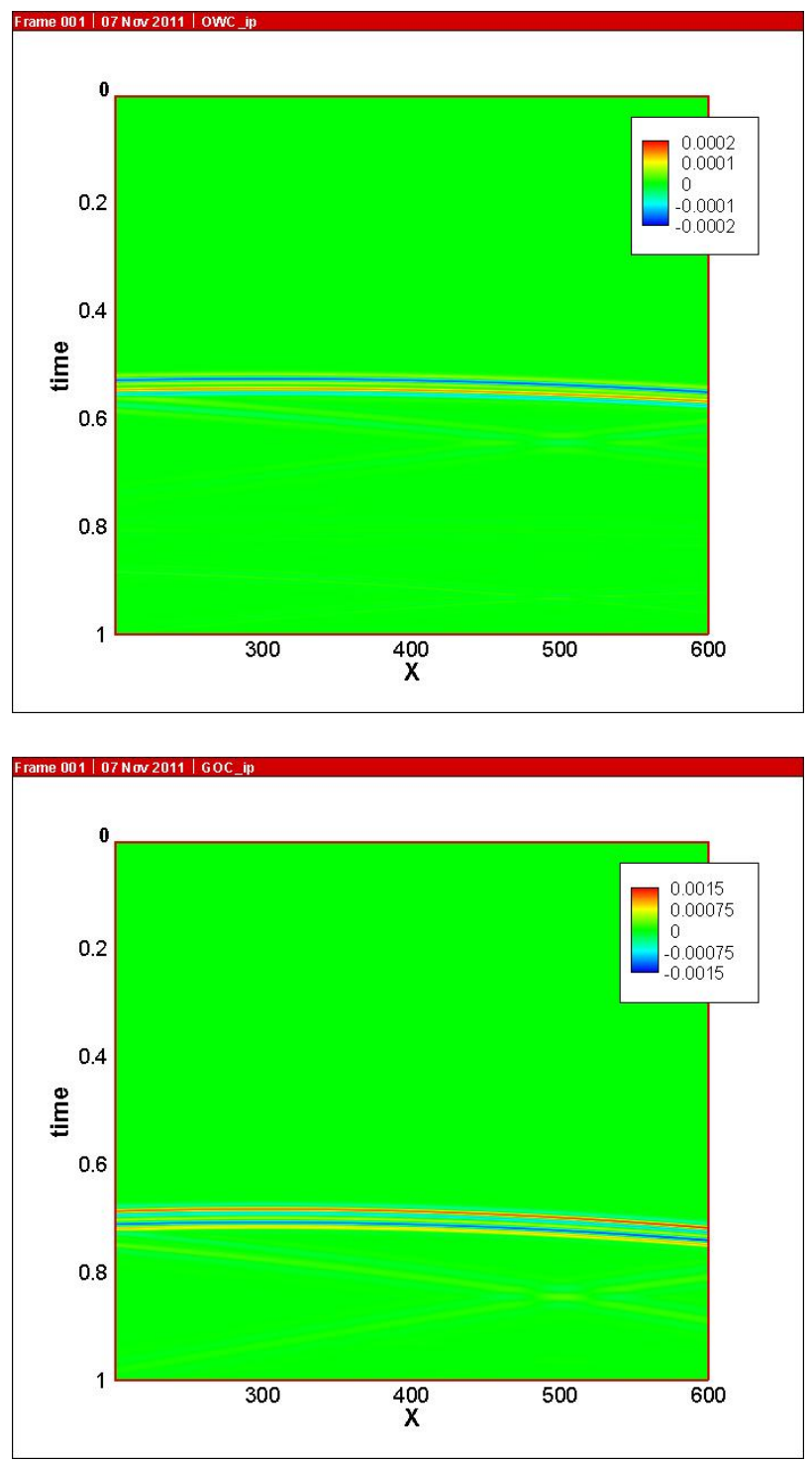

Figure 4 the residual of OWC (upper) and GOC (lower) for partial fluid change with active pore

\section{CONCLUSION}

The movement of GOC provides us information of the abundance and the distribution of oil during the production because of the large difference between oil and gas property. By some simulations for a simple model, we found the residual wavefield that is calculated after the subtraction of the initial wavefield from the simulated after the movement of the contact of two areas. This means that the surface seismic exploration have some possibility to detect the fluid alteration in the reservoir. For the next step, we attempt to specify the space distribution of the GOC and simulate for more complicated model applicable to the reservoir in deep area.

\section{REFERENCES}

1) Biot, M.A., 1956, Theory of propagation of elastic waves in a fluid-saturated porous solid, I - Low-frequency range, Journal of the Acoustic Society of America, 28, 168-178.

2) Biot, M.A., 1962, Mechanics of deformation and acoustic propagation in porous media, Journal of Applied Physics, 33, 1482-1498.

3) Wenzlau, F., Müller, T.M., 2009, Finite-difference modeling of wave propagation and diffusion in poroelastic media, Geophysics, 74, No.4, T55-T66.

4) Endres, A.L., Knight, R.J., 1997, Incorporating pore geometry and fluid pressure communication into modeling the elastic behavior of porous rocks, Geophysics, 62, No.1, 106-117.

5) Kuster, G.T., Toksöz, M.N., 1974, Velocity and attenuation of seismic waves in two-phase media: Part1. theoretical formulations, Geophysics, 39, 587-606.

6) Quinton, W.L., Hayashi, M., Carey, S.K., 2008, Peat hydraulic conductivity in cold regions and its relation to pore size and geometry, Hydrological processes, 22, 2829-2837.

7) Cerjan, C., 1985, A nonreflecting boundary condition for discrete acoustic and elastic wave equations, Geophysics, 50, No.4, 705-708. 